\title{
Myasthenia Classification System
}

National Cancer Institute

\section{Source}

National Cancer Institute. Myasthenia Classification System. NCI Thesaurus. Code

C112013.

A classification system for grading myasthenia gravis that divides the disease into 5 main classes and several subclasses. 